\title{
Transition metal dichalcogenide and hexagonal boron nitride heterostructures grown by molecular beam epitaxy \\ A. T. Barton, ${ }^{1}$ R. Yue, ${ }^{1}$ S. Anwar, ${ }^{1}$ H. Zhu, ${ }^{1}$ X. Peng, ${ }^{1}$ S. McDonnell, ${ }^{1}$ N. Lu, ${ }^{1}$ R. Addou, ${ }^{1}$ \\ L. Colombo ${ }^{2}$, M. J. Kim, ${ }^{1}$ R. M. Wallace, ${ }^{1}$ C. L. Hinkle, ${ }^{1}$ \\ ${ }^{I}$ Materials Science and Engineering, University of Texas at Dallas, Richardson, TX, USA \\ ${ }^{2}$ Texas Instruments, Dallas, TX, USA \\ E-mail address of corresponding author: chris.hinkle@utdallas.edu
}

\section{Abstract}

Heterostructures coupling transition metal dichalcogenides (TMDs) and insulating hexagonal boron nitride (h-BN) were grown by molecular beam epitaxy (MBE) demonstrating the unique opportunities for fabricating all 2D heterostructures (Fig. 1) with the desired band alignments for novel nanoelectronic devices. Structural and chemical characterization of the TMDs and h-BN was conducted via reflection high energy electron diffraction (RHEED), X-ray diffraction (XRD), transmission electron microscopy (TEM), scanning tunneling microscopy/spectroscopy (STM), and X-ray photoelectron spectroscopy (XPS).

\section{Introduction}

Two-dimensional (2D) TMDs are promising materials for future devices to extend the field effect transistor (FET), such as tunnel FETs. TMDs are layered materials similar to graphite that can be metallic or semiconducting depending on the composition.[1] Hexagonal boron nitride (h-BN), a dielectric, has recently emerged as another interesting 2D material for implementation in novel nanoelectronic devices.[2] Because of its wide bandgap, h-BN can be used as a dielectric interlayer for heterostructures coupling layers of graphene or TMDs enabling new all-2D heterostructures. Most of the 2D materials device research to date has been performed

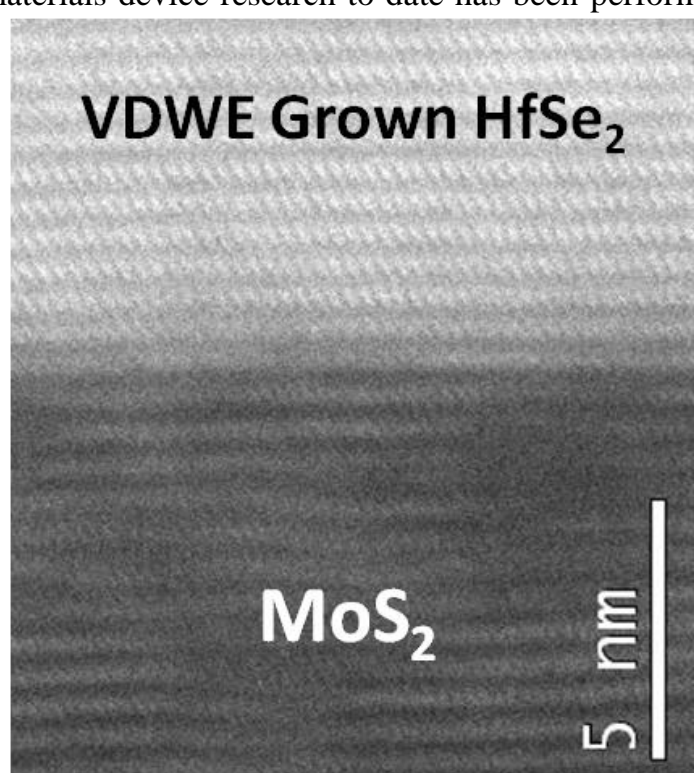

Figure 1. HAADF-STEM image of $\mathrm{HfSe}_{2}$ grown on $\mathrm{MoS}_{2}$ using VDWE in the Hinkle laboratory. using exfoliated, geological TMD materials, such as $\mathrm{MoS}_{2}$. However, these geological TMDs are riddled with native defects[3] (Fig. 2), extrinsic impurities, and damage induced by the violent exfoliation process. Additionally, the exfoliation process results in flake sizes $<1000 \mu \mathrm{m}^{2}$ limiting the research potential of that technique.[4] These issues highlight the critical need for bottom up synthesis of 2D materials to enable large-area, low-defect TMDs and heterostructures. The hexagonal crystal lattices of TMDs and h-BN coupled with van der Waals interactions make them ideal for heterostructure growth without the introduction of extended defects resulting from lattice mismatch as observed in cubic semiconductor materials. Additionally, interdiffusion normally observed in superlattices of II-VI or III-V compounds is reduced in these layered materials. This opens the door for the fabrication of novel nanoelectronics such as vertical broken-gap tunnel FETs and the BiSFET.[5]

\section{Experiments}

TMD and h-BN films were grown on $\sim 1 \times 1 \mathrm{~cm}^{2}$ mechanically exfoliated HOPG, h-BN, and $\mathrm{MoS}_{2}$ substrates purchased from SPI supplies and HQ

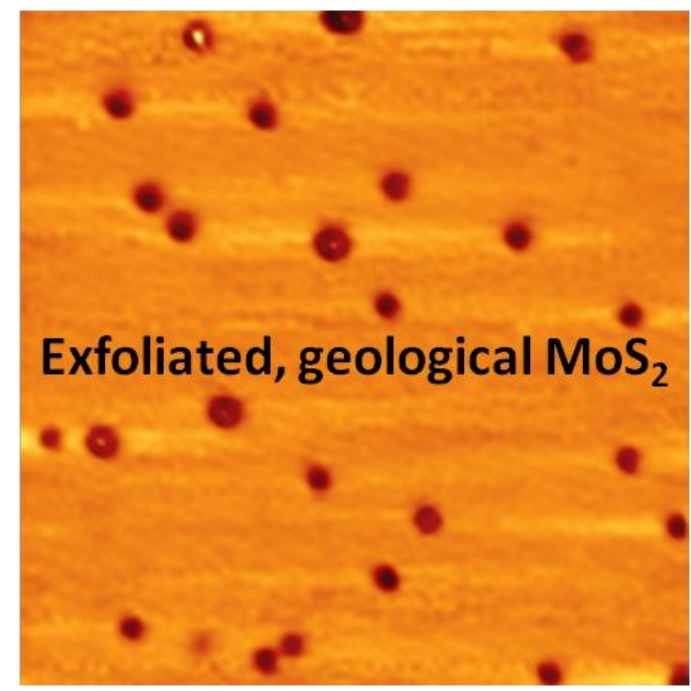

Figure 2. STM image of exfoliated, geological $\mathrm{MoS}_{2}$ demonstrating the high defect density that is common in these samples. These defects and the resultant device variability motivates the need for bottom up growth of TMDs. Image conditions were $100 \mathrm{~nm} \times 100 \mathrm{~nm}, \mathrm{~V}_{\text {bias }}=0.4 \mathrm{~V}, \mathrm{I}_{\mathrm{t}}=1.2 \mathrm{nA}$. 


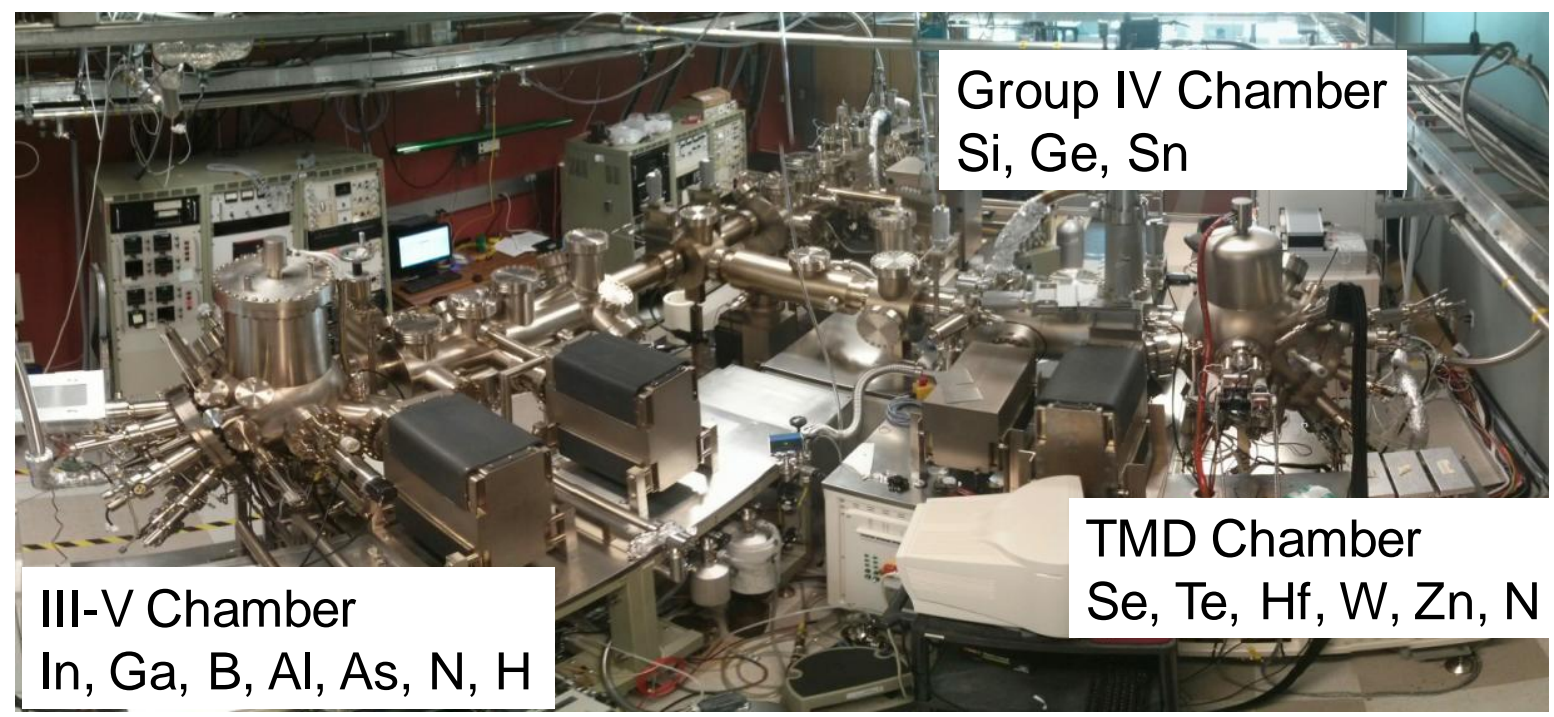

Figure 3. Interconnected multi-chamber MBE system located in the Hinkle laboratory at UT-Dallas.

Graphene. The growth was accomplished in a unique multi-chamber MBE at UT-Dallas. Each of the VGSemicon growth chambers are linked together with a UHV transfer tube system (Fig. 3) operating at a base pressure of about $10^{-11}$ mbar. TMD growth is performed in a $\mathrm{V} 80 \mathrm{H}$ growth chamber equipped with two vertical e-beam evaporators enabling the growth of high melting temperature metals such as $\mathrm{Hf}$, Ti, $\mathrm{Mo}$, and $\mathrm{W}$ in addition to effusion cell evaporation of the chalogens. The two e-beam evaporators allow for TMD heterostructure growth as well as mixed transition metal TMDs, providing significant flexibility in material and device design. Each growth chamber is equipped with in-situ RHEED allowing us to assess the growth quality in real-time. The chamber is also equipped with $\mathrm{ZnCl}_{2}$ and nitrogen plasma sources for doping and surface functionalization.

The III-V chamber is also a V80H with recently upgraded hardware and software to provide state-ofthe-art growth capabilities including In, Ga, As, B, $\mathrm{Al}$, and $\mathrm{N}$ compounds as well as in-situ $\mathrm{H}$-cleaning and $\mathrm{Be}$ and $\mathrm{Si}$ doping. The in-situ growth of hexagonal boron nitride (h-BN) on TMDs. The B is evaporated using a high temperature effusion cell while ultra-high purity $(99.9999 \%) \mathrm{N}_{2}$ gas is used with the Veeco UNI-Bulb RF plasma source that is water cooled and provides outstanding plasma stability. The Group IV chamber, a V80S, is used to grow $\mathrm{Si}, \mathrm{Ge}$, and $\mathrm{Sn}$ epitaxial films, SiSnGe compounds, and strained heterostructures. The vertical growth chamber in this system incorporates electron-beam evaporators and effusion cells for $\mathrm{Sb}$ and B doping. Preparation chambers with high temperature heating stages are available for each material system.

\section{Results and Discussion}

Figure 1 shows a high angle annular dark field (HAADF)-scanning transmission electron microscopy (STEM) image of MBE grown $\mathrm{HfSe}_{2}$ on $\mathrm{MoS}_{2}$, an example of the 2D heterostructures that we have achieved through van der Waals epitaxy (VDWE).[6] We have demonstrated layered films that have atomically abrupt interfaces without misfit dislocations associated with a lattice mismatch as large as 41\%.[7] RHEED data (Fig. 4) shows the starting HOPG (left) and $\sim \mathrm{ML}$ of $\mathrm{HfSe}_{2}$ growth on HOPG (right). The spacing of the reciprocal lattice streaks indicates the growth of unstrained $\mathrm{HfSe}_{2}$ revealing that the TMD immediately has its own lattice constant. This is consistent with TMDs grown by VDWE on other inert hexagonal materials and opens the door for minimized defect heterostructures without concern for substrate lattice matching. The RHEED image also indicates rotational alignment with the hexagonal substrate and flat 2D growth. This rotational alignment may be of critical necessity for some of the advanced device concepts being investigated by enhancing tunneling [8] probability between TMD layers and potentially enabling BoseEinstein Condensate formation.
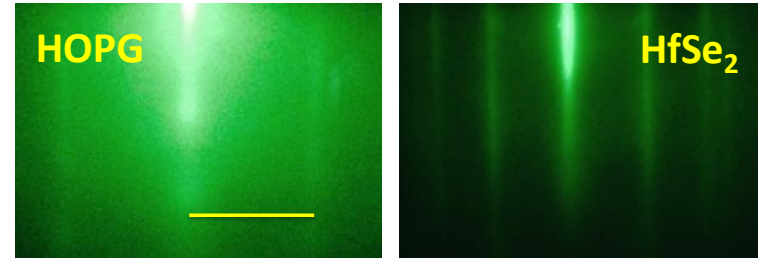

Figure 4. Left: RHEED of starting HOPG substrate. Right: $\sim 1 \mathrm{ML}$ of $\mathrm{HfSe}_{2}$ grown on HOPG. The reciprocal lattice spacing indicates the growth of unstrained $\mathrm{HfSe}_{2}$. 


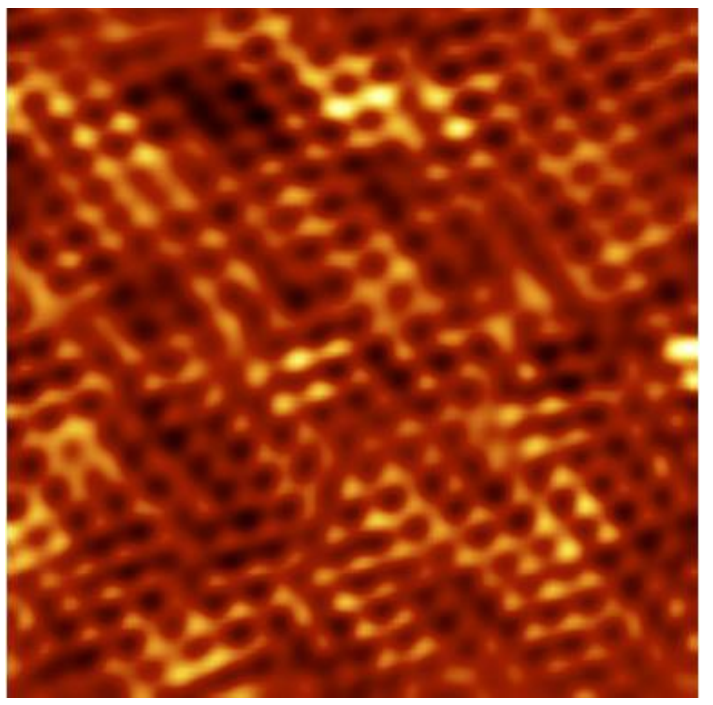

Figure 5. STM image showing atomic resolution of the $\mathrm{MBE}$ grown $\mathrm{HfSe}_{2}$. Image conditions were 5 $\mathrm{nm} \times 5 \mathrm{~nm}, \mathrm{~V}_{\text {bias }}=0.2 \mathrm{~V}, \mathrm{I}_{\mathrm{t}}=2 \mathrm{nA}$.

An STM image of $\mathrm{HfSe}_{2}$ is shown in Figure 5 revealing the hexagonal top surface. The intensity variation observed in the STM image is caused by the onset of surface oxidation of the $\mathrm{HfSe}_{2}$, which occurs much more rapidly that it does on $\mathrm{MoS}_{2}$. While the surface of TMDs is more inert than non-layered materials, surface oxidation is thermodynamically favored for all of them.[9] The difference in the oxidation kinetics of the various TMDs is an interesting field of study that is ongoing.[10] The RHEED, STM, Raman, and XRD analysis (not shown), all indicate that the grown TMDs are unstrained because of the weak van der Waals interactions between the substrate and the growing film.

Figure 6 shows angular-resolved XPS spectra of h-BN grown by MBE on $\mathrm{MoS}_{2}$ indicating that we can successfully grow h-BN on TMDs. XPS can be used to distinguish between $\mathrm{h}-\mathrm{BN}$ and cubic $\mathrm{BN}$ by the presence (or lack thereof) of features at $\sim 200 \mathrm{eV}$ and $408 \mathrm{eV}$. These features are from the $\pi$ plasmon loss features that are indicative of the $\pi$ electrons present in the hexagonal bonded BN.[11] Using the XPS spectra to calculate the stoichiometry shows that our initial h-BN films are slightly $\mathrm{N}$ deficient. We also observe oxidation of the h-BN films after exposure to air which is likely related to non-stoichiometry of the grown films. Figure 7 (left) shows the valence band edge of the h-BN and the onset of the loss feature in Fig. 7 (right) has been used to extract a measured band-gap of $\sim 5 \mathrm{eV}$. The energy separation between the $\mathrm{N} 1 s$ core-level feature and the onset of the associated loss features is used to estimate the h-BN band-gap in a manner analogous to the determination of metal oxide band-gaps using the $\mathrm{O} 1 s$ feature.[1216]. The initial onset of the loss feature is due to band transitions, the minimum energy of which

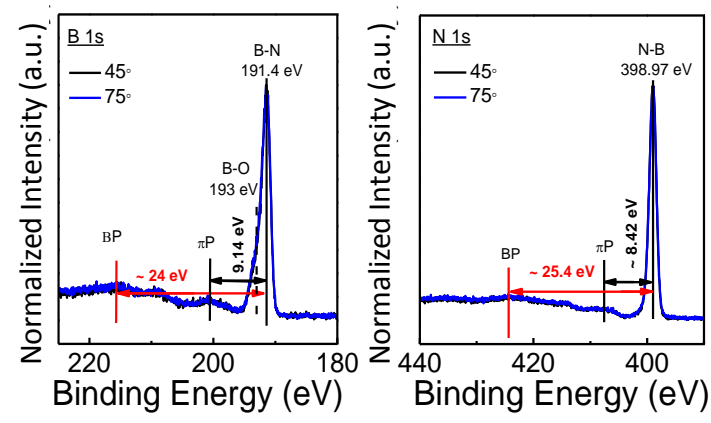

Figure 6. Angular-resolved XPS data from h-BN grown by $\mathrm{MBE}$ on $\mathrm{MoS}_{2}$. The $\pi$ plasmon loss peaks $(\pi \mathrm{P})$ at $\sim 200 \mathrm{eV}$ and $\sim 408 \mathrm{eV}$ indicate hexagonal BN. BP indicates the bulk plasmon loss feature. The signal from the two angles overlaps, indicating uniform chemistry throughout the film.
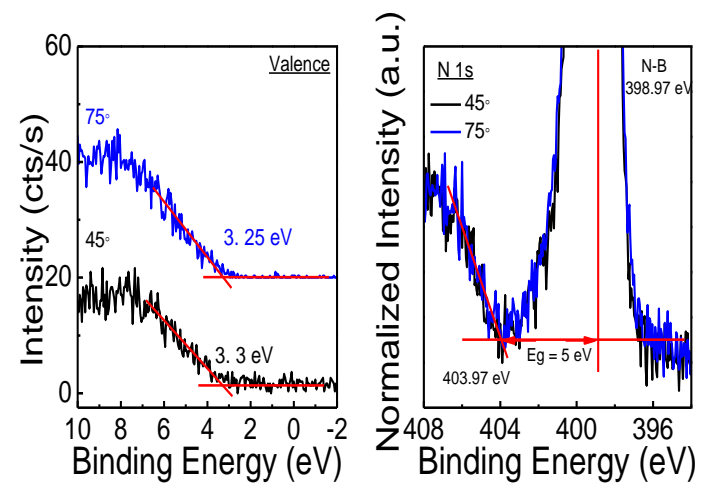

Figure 7. Valence band edge (left) and loss feature (right) used to measure the MBE grown h-BN band-gap.

corresponds to the energy difference between the valence band maximum and the conduction band minimum. $[12,13]$ Therefore, the energy separation of between the $\mathrm{N} 1 s$ core-level and the onset of the loss features can be used to obtain a good estimate of the band-gap of h-BN. These initial h-BN films were sufficiently thick that we could not obtain the valence band offset with the $\mathrm{MoS}_{2}$ due to a lack of signal from the substrate due attenuation.

\section{Summary}

In summary, we have demonstrated the growth of 2D, crystalline TMD and h-BN thin films by MBE and grown heterostructures of these materials. The advantages provided by the van der Waals epitaxy growth method allows the growth of heterostructures with no detectable misfit dislocations or strain in the films despite large lattice mismatch. We have experimentally determined the crystal quality and energy gaps of these materials demonstrating the unique opportunities for fabricating all 2D heterostructures with desirable band alignments that can be useful in the fabrication of future nanoelectronic devices. 


\section{Acknowledgements}

This work is supported in part by the SWAN Center, a SRC center sponsored by the Nanoelectronics Research Initiative and NIST. It is also supported in part by the Center for Low Energy Systems Technology (LEAST), one of six centers of STARnet, a Semiconductor Research Corporation program sponsored by MARCO and DARPA. This work is also supported in part by the Texas Higher Education Coordinating Board's Norman Hackerman Advanced Research Program.

\section{References}

1. Wang, Q. H.; Kalantar-Zadeh, K.; Kis, A.; Coleman, J. N.; Strano, M. S., Electronics and optoelectronics of two-dimensional transition metal dichalcogenides. Nat. Nanotechnol. 7 (11), 699-712,

2012, http://www.nature.com/nnano/journal/v7/n11/abs /nnano.2012.193.html.

2. Song, L.; Ci, L.; Lu, H.; Sorokin, P. B.; Jin, C.; Ni, J.; Kvashnin, A. G.; Kvashnin, D. G.; Lou, J.; Yakobson, B. I., Large scale growth and characterization of atomic hexagonal boron nitride layers. Nano Lett. 10 (8), 3209-3215, 2010.

3. McDonnell, S.; Addou, R.; Buie, C.; Wallace, R. M.; Hinkle, C. L., Defect Dominated Doping and Contact Resistance in $\mathrm{MoS}_{2}$. ACS Nano 8 (3), 2880-2888,

2014, http://pubs.acs.org/doi/abs/10.1021/nn500044q.

4. Li, X. S.; Cai, W. W.; An, J. H.; Kim, S.; Nah, J.; Yang, D. X.; Piner, R.; Velamakanni, A.; Jung, I.; Tutuc, E.; Banerjee, S. K.; Colombo, L.; Ruoff, R. S., Large-Area Synthesis of HighQuality and Uniform Graphene Films on Copper Foils. Science 324 (5932), 1312-1314, 2009, <Go to ISI>://WOS:000266635100041.

5. Banerjee, S. K.; Register, L. F.; Tutuc, E.; Reddy, D.; MacDonald, A. H., Bilayer pseudospin field-effect transistor (BiSFET): a proposed new logic device. IEEE Electron Device Lett. 30 (2), 158-160, 2009, http://dx.doi.org/10.1109/LED.2008.2009362.

6. Ohuchi, F.; Parkinson, B.; Ueno, K.; Koma, A., van der Waals epitaxial growth and characterization of $\mathrm{MoSe}_{2}$ thin films on $\mathrm{SnS}_{2} . J$. Appl. Phys. 68 (5), 2168-2175, 1990.

7. Yue, R.; Barton, A. T.; Zhu, H.; Azcatl, A.; Pena, L. F.; Wang, J.; Peng, X.; Lu, N.; Cheng, L.; Addou, R.; McDonnell, S.; Colombo, L.; Hsu, J. W. P.; Kim, J.; Kim, M. J.; Wallace, R. M.; Hinkle, C. L., HfSe2 Thin Films: 2D Transition Metal Dichalcogenides Grown by Molecular Beam Epitaxy. ACS Nano 9 (1), 474-480, 2014, http://dx.doi.org/10.1021/nn5056496.

8. Lee, K.; Fallahazad, B.; Xue, J.; Dillen, D. C.; Kim, K.; Taniguchi, T.; Watanabe, K.; Tutuc, E., Chemical potential and quantum Hall ferromagnetism in bilayer graphene. Science 345 (6192), 58-61, 2014.

9. Santosh, K. First-Principles Study of Novel Interfaces for Electronic Device and Energy Storage Applications. University of Texas at Dallas, Ph. D. Dissertation, 2014.

10. Azcatl, A.; Santosh KC; Peng, X.; Lu, N.; McDonnell, S.; Qin, X.; De Dios, F.; Addou, R.; Kim, J.; Kim, M. J.; Cho, K.; Wallace, R. M., $\mathrm{HfO}_{2}$ on $\mathrm{UV}-\mathrm{O}_{3}$ exposed transition metal dichalcogenides: interfacial reactions study, $2 D$ Materials, 2, 014004, 2015.

11. Trehan, R.; Lifshitz, Y.; Rabalais, J., Auger and $\mathrm{x}$-ray electron spectroscopy studies of $\mathrm{hBN}, \mathrm{cBN}$, and $\mathrm{N}+2$ ion irradiation of boron and boron nitride. Journal of Vacuum Science \& Technology A 8 (6), 4026-4032, 1990.

12. Bell, F.; Ley, L., Photoemission study of $\mathrm{SiO}_{\mathrm{x}}$ $(0 \leq \mathrm{x} \leq 2)$ alloys. Physical Review B 37 (14), 8383, 1988.

13.Miyazaki, S.; Nishimura, H.; Fukuda, M.; Ley, L.; Ristein, J., Structure and electronic states of ultrathin $\mathrm{SiO}<\mathrm{sub}>2</$ sub $>$ thermally grown on Si (100) and Si (111) surfaces. Appl. Surf. Sci. 113, 585-589, 1997.

14.Miyazaki, S., Photoemission study of energyband alignments and gap-state density distributions for high-k gate dielectrics. Journal of Vacuum Science \& Technology B 19 (6), 22122216, 2001.

15.Ohta, A.; Murakami, H.; Higashi, S.; Miyazaki, S. In Determination of Energy Band Alignment in Ultrathin Hf-based Oxide/Pt System, Journal of Physics: Conference Series, IOP Publishing:; 012012, 2013.

16.McDonnell, S.; Brennan, B.; Azcatl, A.; Lu, N.; Dong, H.; Buie, C.; Kim, J.; Hinkle, C. L.; Kim, M. J.; Wallace, R. M., $\mathrm{HfO}_{2}$ on $\mathrm{MoS}_{2}$ by atomic layer deposition: adsorption mechanisms and thickness scalability. ACS Nano 7 (11), 1035410361, 2013, http://pubs.acs.org/doi/pdfplus/10.1021/nn40477 5u. 


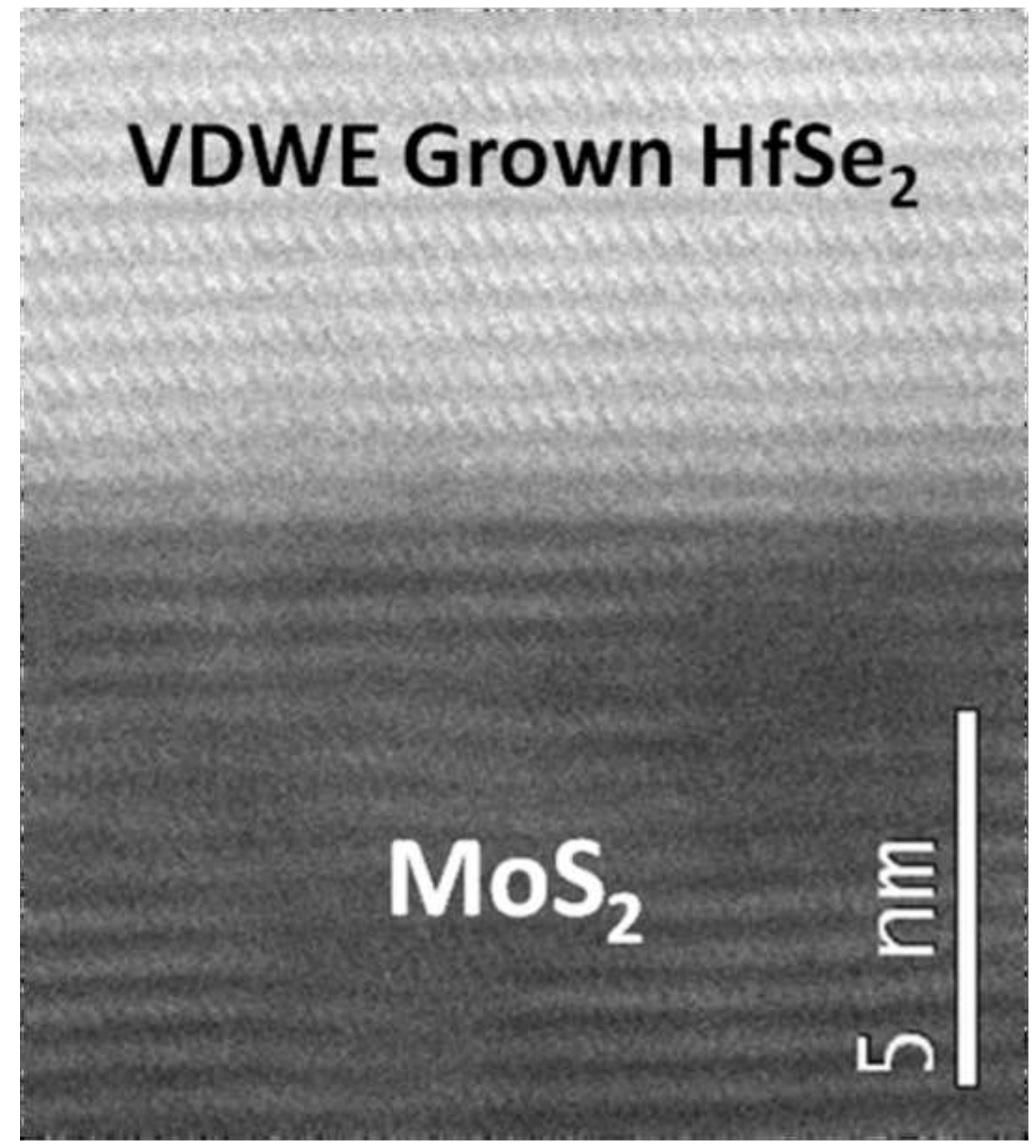

\section{VDWE Grown $\mathrm{HfSe}_{2}$}

\section{*Graphical Abstract}

